Results: 145 patients (46.4\%) were diagnosed with SAR (34 men and 111 women). EN associated with bacterial-viral infection was detected in 167 patients $(53.5 \%)$. Serological examination of these patients showed an increase of $\geq 3$ times the levels of antibodies to Herpesviridae (79 people), M. pneumonie (15), CL. Pneumonia (11), Y. enterocolitica (11), Chl. trachomatis (9), M. hominis (6), increase of ASL-O (57). EN with SAR is characterized by: the duration of the disease up to 6 months (odds ratio (OR) 7,320, 95\% confidence interval (Cl) 2,297- 23,329, $\mathrm{p}<0.005)$, multiple diffuse lesions on the legs and single diffuse lesions on the thighs and/or forearms (OR 15,500, 95\% Cl 1,983-121,132; $\mathrm{p}<0.005$ ), lesions of ankle joints (OSH 35,905, 95\% Cl 9,523-135,365; $\mathrm{p}<0.005$ ), high CRP level (OR 5,429 95\% Cl: 2,164-13,618; $\mathrm{p}<0.005$ ). Predictors of the formation of the CT phenomenon of "frosted glass" in ATS were male sex (OR 6.5; DI 1.2-35; $p=0.026$ ) and the presence of conglomerates of nodes (OR 4.8; DI 1.4-16.1; $p=0.01$ ). Postinfectious EN was characterized by its development typically before the age of 30 (OR $0.825,95 \% \mathrm{Cl} 0.328-2.077, \mathrm{p}<0.005$ ), the symptom of "blooming" bruise (OR $0.028,95 \% \mathrm{Cl} 0.009-0.092, \mathrm{p}<0.005)$, an increase in antistreptolysin-O (OR $0.035,95 \%$ Cl $0.006-0.207, \quad \mathrm{p}<0.005)$. Within 1 year of follow-up, $72 \%$ of patients with SAR EN did not relapse, and the joint syndrome almost completely regressed. Postinfectious EN recurred within 1 year in 38 people due to: exacerbation of chronic tonsillitis (14), ARVI/hypothermia (10), stress (5), violation and ineffectiveness of prescribed therapy (9).

Conclusion: Post-infectious EN is somewhat more common than EN in SAR. The latter is characterized by greater severity of clinical parameters and high parameters of inflammatory activity. These features should be borne in mind when carrying out differential diagnosis in patients with EN.

Disclosure of Interests: None declared

DOI: 10.1136/annrheumdis-2019-eular.3736

\section{AB1085 OSTEO-ARTICULAR MANIFESTATIONS OF SARCOIDOSIS: ABOUT 13 CASES}

ACHEK Mohamed Amine EL, Khadija Baccouche, Amel Farhat, Nejla El Amri, Hela Zeglaoui, Elyes Bouajina. Farhat Hached University Hospital, Rheumatology, Sousse, Tunisia

Background: Sarcoidosis is a systemic disorder of unknown etiology, affecting the respiratory tract and the lymphatic system with predilection It is an ubiquitous condition whose clinical phenotype is very diverse.

Objectives: To detail the epidemiological, clinico-biological, therapeutic and evolutionary features of osteoarticular forms of sarcoidosis.

Methods: This is a monocentric, descriptive retrospective study conducted over a 17-years period [2002-2018] involving 13 patients diagnosed with sarcoidosis with osteoarticular manifestations at the department of Rheumatology at Farhat Hached University Hospital in Sousse.

Results: 13 patients with osteoarticular manifestations were studied; this was indicative of the disease in 8 cases $(61.5 \%)$. The sex ratio was 0.23 with a mean age of 42.38 years and a diagnostic delay of 12 months. In $77 \%$ of cases, inflammatory arthralgia was noted, Löfgren syndrome $(30.7 \%)$, chronic polyarthritis $(30.7 \%)$, phalanges cystoid osteitis $(15.4 \%)$ and no cases of monoarthritis. The extra articular manifestations found were; Adenomegaly (92.3\%), lung (77\%), cutaneous (38.5\%) (including $80 \%$ erythema nodosum), neurological (30.7\%) (Aseptic meningitis and convulsion), ocular (23\%) (chorioretinitis, keratoconjunctivitis and retro-bulbar optic neuritis), cardiac (15.4\%), and biological hepatitis $(7.7 \%)$. In the biology, a biological inflammatory syndrome was found in $30.7 \%$ with a mean sedimentation rate of 31.07 in the 1 st hour, an average CRP of $9 \mathrm{mg} / \mathrm{L}$, cholestasis and cytolysis found in $15.4 \%$ and $7.7 \%$, respectively. No Hypercalcemia was noted. The value of the conversion enzyme was high in 5 out of 6 patients who were dosed with an average of $71 \mu \mathrm{g} / \mathrm{L}$. In imaging, sarcoidosis was destructive in 4 cases; A multifocal osteonecrosis, Algodystrophy of the two hips and two cases of bullous cystoid osteitis of phalanges.

Conclusion: The osteo-articular involvement of sarcoidosis is polymorphic, which can reveal the disease or enamel its evolution.

Disclosure of Interests: None declared

DOI: 10.1136/annrheumdis-2019-eular. 6434

\section{AB1086 \\ ADULT ONSET STILL'S DISEASE: EXPERIENCE OF THE RHEUMATOLOGY DEPARTMENT OF THE UNIVERSITY HOSPITAL OF CASABLANCA}

Zakaria El Ouali, Saadia Janani, Kawtar Nassar, Ouafa Mkinsi. CHU Ibn Rochd, Department of Rheumatology, Casablanca, Morocco

Background: Adult onset Still's disease (AOSD) is a rare condition characterized by four cardinal signs: fever, arthralgia/arthritis, rash, and neutrophilic leukocytosis. The absence of specific criteria is currently compensated by pathophysiological advances, leading to the introduction of a promising new therapeutic arsenal represented by biotherapies.

Objectives: The aim of this study was to compare the profiles of this disease in our latitudes with data from foreign literature; and to study the most recent data concerning the pathophysiology and treatment of this disease.

Methods: We conducted a retrospective study of hospital files from January 1993 to October 2018. The diagnosis is retained according to Yamaguchi criteria. Analysis was performed using IPSS.

Results: 17 patients were selected. The sex ratio was 0.54 , and the average age was 31.5 years. At the time of diagnosis, fever was found in $94 \%$ of cases, arthralgia/arthritis in $100 \%$ of cases, and rash in $65 \%$ of cases. Concerning rheumatic features, the most frequently affected joints were: wrists $(87 \%)$, knees $(73 \%)$, shoulders $(67 \%)$, proximal interphalangeal $(62 \%)$, elbows and ankles $(60 \%)$, hips $(47 \%)$. The involvement of the hands is dominated by the proximal interphalangeal and metacarpophalangeal $(45 \%)$. Distal interphalangeal involvement is observed in $23 \%$ of cases. Cervical spine involvement is noted in $50 \%$ of cases. Biologically, $88 \%$ of patients had neutrophilic leukocytosis. Ferritinemia was increased in $79 \%$ of patients. The glycosylated fraction of ferritin was measured at diagnosis in $47 \%$ of patients, of whom $63 \%$ had a fraction $\leq 20 \%$. Elevated transaminases were found in $47 \%$ of cases. Therapeutically, $100 \%$ of patients received corticosteroids; $65 \%$ methotrexate; and $29 \%$ biotherapies (tocilizumab, anakinra, infliximab, adalimumab). The evolutionary profile was exploitable in 13 patients, of whom 10 were in complete remission.

Conclusion: Our data are consistent with those in the literature. Some peculiarities emerge concerning the articular features. From 3 series of literature and ours, totaling 135 cases, it appears that the most frequent locations are: wrist $(82 \%)$, knees $(70 \%)$, elbows $(46 \%)$, ankles $(45 \%)$, proximal interphalangeal (44\%), shoulders (42\%) and metacarpophalangeal $(42 \%)$. The pathogenesis of AOSD seems to lie at the crossroads between auto-inflammatory diseases and lymphohistiocyte activation, resulting from a multifactorial predisposition. The central role of innate immunity, the clinical presentation, and the efficacy of blocking the IL-1 pathway comparable to that observed in "monogenic inflammasomopathies" justify the reclassification of AOSD among polygenic auto-inflammatory syndromes.

\section{REFERENCES}

[1] Georgin-Lavialle S, et al. [Clinical overview of auto-inflammatory diseases]. Rev Med Interne. 2018;39(4):214-32.

[2] Gerfaud-Valentin $\mathrm{M}$, et al. Adult-onset still disease: manifestations, treatment, outcome, and prognostic factors in 57 patients. Medicine (Baltimore) 2014;93(2):91-9.

[3] Wouters JM, et al. Adult-onset Still's disease; clinical and laboratory features, treatment and progress of 45 cases. Q J Med. 1986;61(235):1055 65 .

[4] Pouchot J, et al. Adult Still's disease: manifestations, disease course, and outcome in 62 patients. Medicine (Baltimore). 1991;70(2):118-36.

[5] Ben Taarit C, et al.[Adult Still's disease: study of a series of 11 cases]. J Mal Vasc. 2002;27(1):31-5.

Disclosure of Interests: None declared

DOI: 10.1136/annrheumdis-2019-eular.2204 